\title{
Self-injection locking of a laser diode to a high-Q silicon WGM microresonator
}

\author{
Artem Shitikov ${ }^{1,2, *}$, Tatyana Tebeneva ${ }^{3}$, Nikita Kondratiev ${ }^{1}$, Valery Lobanov ${ }^{1}$, \\ Oleg Benderov ${ }^{3}$, Alexander Rodin ${ }^{3}$, and Igor Bilenko ${ }^{1,2}$ \\ ${ }^{1}$ Russian Quantum Center, 121205 Moscow, Russia \\ ${ }^{2}$ Lomonosov MSU, Faculty of Physics, 119991 Moscow, Russia \\ ${ }^{3}$ Moscow institute of physics and technologies, 141701 Dolgoprudny, Moscow Region, Russia
}

\begin{abstract}
The key properties of the self-injection locking regime of a laser diode to a high-Q microresonator with whispering gallery mode made of crystalline silicon are considered. It has been experimentally demonstrated the possibility of the self-injection locking using cavity made of crystalline silicon. This result opens up new possibilities for creating narrow-band highly stable laser sources in midIR, over 2.3 microns, on a new hardware base.
\end{abstract}

One of the promising ways to stabilize a laser diode is to use the self-injection locking regime of a laser diode to a high-Q whispering gallery mode (WGM) microcavity [1]. A feature of this approach is that the microcavity provides frequency-selective feedback, which allows one to combine the high radiation power of the laser diode with a narrow linewidth. It is worth noting, that one may use the technique to obtain narrow-band single-frequency generation using a multi-frequency laser diode [2]. A laser linewidth of the order of several Hzs may be achieved using this technique (and even less [3]). The implementation of the self-injection locking in midIR is rather challenging and important task. The multiphonon absorption is a dominant factor in widely used fluorides in midIR from 4 um [4], in contrast the multiphonon absorption in silicon is negligible up to $6.5 \mathrm{um}$.

Silicon is a dielectric material where strong two-photon absorption is observed up to 2.3 microns. Until recently, the applicability of silicon microresonators in optics and photonics was limited by the relatively low quality factor of cavities, which, as shown by further studies, could be associated with non-optimal surface treatment processes. In a recent work [5], a record billion Q-factor of silicon at a wavelength of $1550 \mathrm{~nm}$ was demonstrated. We note that silicon is a material with an extremely high coefficient of cubic nonlinearity of $n_{2}=4,5 \cdot 10^{-18} \mathrm{~m}^{2} / \mathrm{W}$, which opens up particular prospects for the generation of optical frequency combs in it. The use of the scheme proposed in $[6,7]$, paves the way to the generation of coherent optical frequency combs in midIR under the self-injection locking.

\footnotetext{
* Corresponding author: Shartev@gmail.com
} 

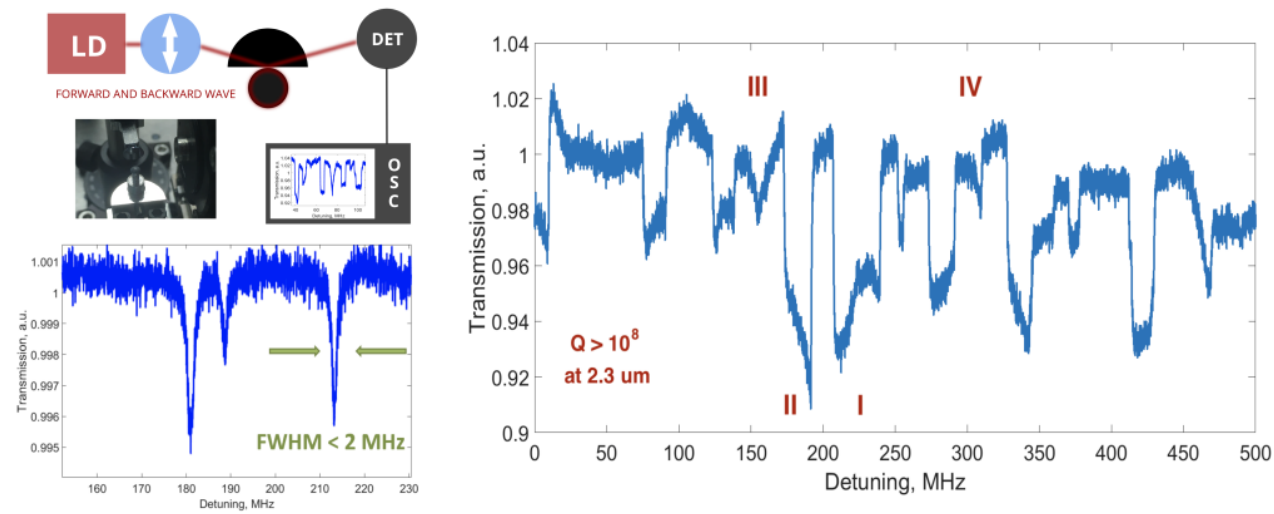

Fig. 1. Top left is a sketch of an experimental setup. Bottom left is the spectrum of WGM modes in the undercoupled regime. The quality factor of the resonator is determined at half maximum of the line. On the right is a mode spectrum of a laser diode in the self-injection locking regime.

In our work, we experimentally investigated the possibility of using silicon microcavities to stabilize laser diodes operating at a wavelength of $2.3 \mu \mathrm{m}$. The experimental setup is shown in Fig. 1 (top left). We used a resonator made of crystalline silicon obtained by the Czochralski method with a resistivity of $6 \mathrm{k} \Omega$. The microresonator was fabricated according to the technique described in [5]. The diameter of the resonator is $2.5 \mathrm{~mm}$, the radius of curvature is $1.5 \pm 0.5 \mathrm{~mm}$, and the thickness is $1 \mathrm{~mm}$. The resonator $\mathrm{Q}$ factor at a wavelength of $1.55 \mu \mathrm{m}$ exceeded $2 \times 10^{8}$ (Fig. 1 bottom left). A 2.3-micron distributed-feedback laser was used as a signal source for the WGM excitation. The laser and the resonator were located at a distance of $60 \mathrm{~cm}$ from each other. A silicon hemisphere served as an element of communication with the resonator for exciting whispering gallery modes with the angle of incidence $85.5^{\circ}$.

We proved experimentally that self-injection locking regime may be achieved for WGM microresonators made of crystalline silicon by means of hemispherical silicon coupler. The self-injection locking range reached $50 \mathrm{MHz}$ for the critical coupling. By adjusting the distance between the laser and the microresonator, one may adjust the phase shift of the backward wave, and switch between the self-injection locking regimes (Fig. 1 on the right) - the regions in which the laser frequency practically does not change under the current tuning. Four different modes are distinguished depending on the different phase shifts of the backward wave $\psi_{0}=[0, \pi / 2, \pi, 3 \pi / 2]$, while the regime with the zero-phase shift is optimal. We also estimated the Q at $2.3 \mu \mathrm{m}$ by the reduce of the back scattering, it exceeded $10^{8}$.

The work was supported by Russian Science Foundation (project 17-12-01413).

\section{References}

1. N.M. Kondratiev, V.E. Lobanov, A.V. Cherenkov et al., Optics Express 25, 28167 (2017)

2. R.R. Galiev, N.G. Pavlov, N.M. Kondratiev et al., Opt. Express 26, 30509 (2018)

3. W. Liang, V.S. Ilchenko, D. Eliyahu et al., Nature Comm 6, 7371 (2015)

4. C. Lecaplain, C. Javerzac-Galy, M.L. Gorodetsky et al., Nat. Commun 7, 1383 (2016)

5. A.E. Shitikov, I.A. Bilenko, N.M. Kondratiev et al., Optica 5, 1525 (2018)

6. N. G. Pavlov, S. Koptyaev, G.V. Lihachev et al., Nat. Photon. 12, 694 (2018)

7. A.S. Raja, A.S. Voloshin, H. Guo et al., Nature Communications. 10, 680 (2019) 